PROCEEDINGS OF THE

AMERICAN MATHEMATICAL SOCIETY

Volume 136, Number 10, October 2008, Pages 3571-3577

S 0002-9939(08)09569-5

Article electronically published on June 6, 2008

\title{
ON A CLASS OF IDEALS OF THE TOEPLITZ ALGEBRA ON THE BERGMAN SPACE
}

\author{
TRIEU LE
}

(Communicated by Marius Junge)

\begin{abstract}
Let $\mathfrak{T}$ denote the full Toeplitz algebra on the Bergman space of the unit ball $\mathbb{B}_{n}$. For each subset $G$ of $L^{\infty}$, let $\mathfrak{C I}(G)$ denote the closed twosided ideal of $\mathfrak{T}$ generated by all $T_{f} T_{g}-T_{g} T_{f}$ with $f, g \in G$. It is known that $\mathfrak{C I}\left(C\left(\overline{\mathbb{B}}_{n}\right)\right)=\mathcal{K}$, the ideal of compact operators, and $\mathfrak{C} \mathfrak{I}\left(C\left(\mathbb{B}_{n}\right) \cap L^{\infty}\right)=\mathfrak{T}$. Despite these "extreme cases", there are subsets $G$ of $L^{\infty}$ so that $\mathcal{K} \subsetneq \mathfrak{C} \mathfrak{I}(G) \subsetneq$ $\mathfrak{T}$. This paper gives a construction of a class of such subsets.
\end{abstract}

\section{INTRODUCTION}

For any integer $n \geq 1$, let $\mathbb{C}^{n}$ denote the Cartesian product of $n$ copies of $\mathbb{C}$. For $z=\left(z_{1}, \ldots, z_{n}\right)$ and $w=\left(w_{1}, \ldots, w_{n}\right)$ in $\mathbb{C}^{n}$, we write $\langle z, w\rangle=z_{1} \bar{w}_{1}+\cdots+z_{n} \bar{w}_{n}$ and $|z|=\sqrt{\left|z_{1}\right|^{2}+\cdots+\left|z_{n}\right|^{2}}$ for the inner product and the associated Euclidean norm. Let $\mathbb{B}_{n}$ denote the open unit ball which consists of all $z \in \mathbb{C}^{n}$ with $|z|<1$. Let $\mathbb{S}_{n}$ denote the unit sphere which consists of all $z \in \mathbb{C}^{n}$ with $|z|=1$. For any subset $V$ of $\mathbb{B}_{n}$ we write $\operatorname{cl}(V)$ for the closure of $V$ as a subset of $\mathbb{C}^{n}$ with respect to the Euclidean metric. We write $\overline{\mathbb{B}}_{n}$ for the closed unit ball which is also $\mathrm{cl}\left(\mathbb{B}_{n}\right)$. Let $C\left(\mathbb{B}_{n}\right)$ (respectively, $C\left(\overline{\mathbb{B}}_{n}\right)$ ) denote the space of all functions that are continuous in the open unit ball (respectively, the closed unit ball).

Let $\nu$ denote the Lebesgue measure on $\mathbb{B}_{n}$ normalized so that $\nu\left(\mathbb{B}_{n}\right)=1$. Let $L^{2}=L^{2}\left(\mathbb{B}_{n}, \mathrm{~d} \nu\right)$ and $L^{\infty}=L^{\infty}\left(\mathbb{B}_{n}, \mathrm{~d} \nu\right)$. The Bergman space $L_{a}^{2}$ is the subspace of $L^{2}$ which consists of all analytic functions. The normalized reproducing kernels for $L_{a}^{2}$ are of the form

$$
k_{z}(w)=\left(1-|z|^{2}\right)^{(n+1) / 2}(1-\langle w, z\rangle)^{-n-1},|z|,|w|<1 .
$$

We have $\left\|k_{z}\right\|=1$ and $\left\langle g, k_{z}\right\rangle=\left(1-|z|^{2}\right)^{(n+1) / 2} g(z)$ for all $g \in L_{a}^{2}, z \in \mathbb{B}_{n}$.

The orthogonal projection from $L^{2}$ onto $L_{a}^{2}$ is given by

$$
(P g)(z)=\int_{\mathbb{B}_{n}} \frac{g(w)}{(1-\langle z, w\rangle)^{n+1}} \mathrm{~d} \nu(w), g \in L^{2}, z \in \mathbb{B}_{n} .
$$

For any $f \in L^{\infty}$, the Toeplitz operator $T_{f}: L_{a}^{2} \longrightarrow L_{a}^{2}$ is defined by $T_{f} h=P(f h)$ for $h \in L_{a}^{2}$. We have

$$
\left(T_{f} h\right)(z)=\int_{\mathbb{B}_{n}} \frac{f(w) h(w)}{(1-\langle z, w\rangle)^{n+1}} \mathrm{~d} \nu(w)
$$

Received by the editors August 16, 2007.

2000 Mathematics Subject Classification. Primary 47B35; Secondary 47B47.

(C)2008 American Mathematical Society 3571 Reverts to public domain 28 years from publication 
for $h \in L_{a}^{2}$ and $z \in \mathbb{B}_{n}$.

For all $f \in L^{\infty},\left\|T_{f}\right\| \leq\|f\|_{\infty}$ and $T_{f}^{*}=T_{\bar{f}}$. In contrast with Toeplitz operators on the Hardy space of the unit sphere, there are functions $f \in L^{\infty}$ so that $\left\|T_{f}\right\|<$ $\|f\|_{\infty}$. Since $T_{f}$ is an integral operator by equation (1.1), we see that $T_{f}$ is compact if $f$ vanishes almost everywhere in the complement of a compact subset of $\mathbb{B}_{n}$.

Let $\mathfrak{B}\left(L_{a}^{2}\right)$ be the $\mathrm{C}^{*}$-algebra of all bounded linear operators on $L_{a}^{2}$. Let $\mathcal{K}$ denote the ideal of $\mathfrak{B}\left(L_{a}^{2}\right)$ that consists of all compact operators. The full Toeplitz algebra $\mathfrak{T}$ is the $\mathrm{C}^{*}$-subalgebra of $\mathfrak{B}\left(L_{a}^{2}\right)$ generated by $\left\{T_{f}: f \in L^{\infty}\right\}$. For any subset $G$ of $L^{\infty}$, let $\mathfrak{I}(G)$ denote the closed two-sided ideal of $\mathfrak{T}$ generated by all $T_{f}$ with $f \in G$. Let $\mathfrak{C} \mathfrak{I}(G)$ denote the closed two-sided ideal of $\mathfrak{T}$ generated by all commutators $\left[T_{f}, T_{g}\right]=T_{f} T_{g}-T_{g} T_{f}$ with $f, g \in G$. A result of L. Coburn [1] in the 1970's showed that $\mathfrak{C I}\left(C\left(\overline{\mathbb{B}}_{n}\right)\right)=\mathcal{K}$. In 2004, D. Suárez [5] showed that $\mathfrak{C} \mathfrak{I}\left(L^{\infty}\right)=\mathfrak{T}$ for the case $n=1$. This result has been generalized by the author 2 ] to all $n \geq 1$. In fact, we are able to show that $\mathfrak{C} \mathfrak{I}(G)=\mathfrak{T}$ for certain subsets $G$ of $L^{\infty}$. We can take $G=\left\{f \in C\left(\mathbb{B}_{n}\right) \cap L^{\infty}: f\right.$ vanishes on $\left.\mathbb{B}_{n} \backslash E\right\}$, where $0<\nu(E)$ can be as small as we please. We can also take $G=\left\{f \in L^{\infty}: f\right.$ vanishes on $\left.\mathbb{B}_{n} \backslash E\right\}$ where $E$ is a closed nowhere dense subset of $\mathbb{B}_{n}$ with $0<\nu(E)$ as small as we please. From these results, one may be interested in the question: is there any subset $G$ of $L^{\infty}$ so that $\mathcal{K} \subsetneq \mathfrak{C} \mathfrak{I}(G) \subsetneq \mathfrak{T}$ ? The purpose of this paper is to show that there are infinitely many such subsets. Our main result is the following theorem.

Theorem 1.1. To every closed subset $F$ of $\mathbb{S}_{n}$, there is a subset $G_{F}$ of $L^{\infty}$ so that the following statements hold true:

(1) $\mathfrak{I}\left(G_{\emptyset}\right)=\mathcal{K}$ and $\mathfrak{C} \mathfrak{I}\left(G_{\mathbb{S}_{n}} \cap C\left(\mathbb{B}_{n}\right)\right)=\mathfrak{T}$.

(2) If $F_{1}, F_{2}$ are closed subsets of $\mathbb{S}_{n}$ and $F_{1} \subset F_{2}$, then $G_{F_{1}} \subset G_{F_{2}}$.

(3) If $F_{1}, F_{2}$ are closed subsets of $\mathbb{S}_{n}$ and $F_{2} \backslash F_{1} \neq \emptyset$, then we have $\mathfrak{C} \mathfrak{I}\left(G_{F_{2}} \cap\right.$ $\left.C\left(\mathbb{B}_{n}\right)\right) \backslash \mathfrak{I}\left(G_{F_{1}}\right) \neq \emptyset$. In particular, if $\emptyset \neq F \subsetneq \mathbb{S}_{n}$, then $\mathcal{K} \subsetneq \mathfrak{C I}\left(G_{F} \cap\right.$ $\left.C\left(\mathbb{B}_{n}\right)\right) \subset \mathfrak{I}\left(G_{F}\right) \subsetneq \mathfrak{T}$.

In Section 2 and Section 3 we provide some preliminaries and basic results. In Section 4, we give a proof for Theorem 1.1 .

\section{Preliminaries}

For any $z \in \mathbb{B}_{n}$, let $\varphi_{z}$ denote the Mobius automorphism of $\mathbb{B}_{n}$ that interchanges 0 and $z$. For any $z, w \in \mathbb{B}_{n}$, let $\rho(z, w)=\left|\varphi_{z}(w)\right|$. Then $\rho$ is a metric on $\mathbb{B}_{n}$ (called the pseudo-hyperbolic metric) which is invariant under the action of the automorphism group $\operatorname{Aut}\left(\mathbb{B}_{n}\right)$ of $\mathbb{B}_{n}$. For any $w \in \mathbb{B}_{n}$, we have $\rho(z, w) \rightarrow 1$ as $|z| \rightarrow 1$. These properties and the following inequality can be proved by using the identities in [4, Theorem 2.2.2]. See [2, Section 2] for more details.

For any $z, w, u \in \mathbb{B}_{n}$,

$$
\rho(z, w) \leq \frac{\rho(z, u)+\rho(u, w)}{1+\rho(z, u) \rho(u, w)} .
$$

For any $a$ in $\mathbb{B}_{n}$ and any $0<r<1$, let $B(a, r)=\left\{z \in \mathbb{B}_{n}:|z-a|<r\right\}$ and $E(a, r)=\left\{z \in \mathbb{B}_{n}: \rho(z, a)<r\right\}$. 
Inequality (2.1) shows that if $z, w \in \mathbb{B}_{n}$ so that $E\left(z, r_{1}\right) \cap E\left(w, r_{2}\right) \neq \emptyset$ for some $0<r_{1}, r_{2}<1$, then

$$
\begin{aligned}
\rho(z, w) & \leq \frac{\rho(z, u)+\rho(u, w)}{1+\rho(z, u) \rho(u, w)} \quad\left(\text { where } u \text { is any element in } E\left(z, r_{1}\right) \cap E\left(w, r_{2}\right)\right) \\
& <\frac{r_{1}+r_{2}}{1+r_{1} r_{2}} .
\end{aligned}
$$

This implies that if $\rho(z, w) \geq \frac{r_{1}+r_{2}}{1+r_{1} r_{2}}$, then $E\left(z, r_{1}\right) \cap E\left(w, r_{2}\right)=\emptyset$.

Lemma 2.1. For any $0<r<1$ and $\zeta \in \mathbb{S}_{n}$ there is an increasing sequence $\left\{t_{m}\right\}_{m=1}^{\infty} \subset(0,1)$ so that $t_{m} \rightarrow 1$ as $m \rightarrow \infty$ and $E\left(t_{k} \zeta, r\right) \cap E\left(t_{l} \zeta, r\right)=\emptyset$ for all $k \neq l$.

Proof. We will construct the required sequence $\left\{t_{m}\right\}_{m=1}^{\infty}$ by induction. We begin by taking any $t_{1}$ in $(0,1)$. Suppose we have chosen $t_{1}<\cdots<t_{m}$ so that $1-j^{-1}<$ $t_{j}<1$ for all $1 \leq j \leq m$ and $E\left(t_{k} \zeta, r\right) \cap E\left(t_{l} \zeta, r\right)=\emptyset$ for all $1 \leq k<l \leq m$, where $m \geq 1$. Since $\rho\left(t \zeta, t_{j} \zeta\right) \rightarrow 1$ as $t \uparrow 1$ for all $1 \leq j \leq m$, we can choose a $t_{m+1}$ with $\max \left\{t_{m}, 1-(m+1)^{-1}\right\}<t_{m+1}<1$ and $\rho\left(t_{m+1} \zeta, t_{j} \zeta\right)>\frac{2 r}{1+r^{2}}$ for all $1 \leq j \leq m$. It then follows that $E\left(t_{m+1} \zeta, r\right) \cap E\left(t_{j} \zeta, r\right)=\emptyset$ for all $1 \leq j \leq m$. Also, since $1-m^{-1}<t_{m}<1$ for all $m, t_{m} \rightarrow 1$ as $m \rightarrow \infty$.

Lemma 2.2. For any $0<r<1$ and any $\epsilon>0$, there is a $\delta$ depending on $r$ and $\epsilon$ so that for all $\zeta \in \mathbb{S}_{n}$ and all $a \in \mathbb{B}_{n}$ with $|a-\zeta|<\delta$, we have

$$
E(a, r) \subset\left\{z \in \mathbb{B}_{n}:|z-\zeta|<\epsilon\right\} .
$$

As a consequence, if $b \in \mathbb{B}_{n}$ and $\zeta \in \mathbb{S}_{n}$ so that $E(b, r) \cap\left\{z \in \mathbb{B}_{n}:|z-\zeta|<\delta\right\} \neq \emptyset$, then $|b-\zeta|<\epsilon$.

Proof. From [4, Section 2.2.7], for any $a \neq 0$,

$$
E(a, r) \subset\left\{z \in \mathbb{B}_{n}: \frac{|P z-c|^{2}}{r^{2} s^{2}}+\frac{|Q z|^{2}}{r^{2} s}<1\right\}
$$

where $P z=\frac{\langle z, a\rangle}{\langle a, a\rangle} a, Q z=z-P z, c=\frac{1-r^{2}}{1-r^{2}|a|^{2}} a$ and $s=\frac{1-|a|^{2}}{1-r^{2}|a|^{2}}$. It then follows that $E(a, r) \subset B(c, r \sqrt{s})$.

Since $|a-c|=r^{2} s|a| \leq r \sqrt{s}$, we get $B(c, r \sqrt{s}) \subset B(a, 2 r \sqrt{s})$. Hence $E(a, r) \subset$ $B(a, 2 r \sqrt{s})$. Note that the inclusion certainly holds true for $a=0$ (in this case $s=1)$.

Now suppose $|a-\zeta|<\delta$. Then $|a| \geq|\zeta|-|a-\zeta|>1-\delta$. Hence,

$$
s=\frac{1-|a|^{2}}{1-r^{2}|a|^{2}} \leq \frac{1-|a|^{2}}{1-r^{2}} \leq \frac{2(1-|a|)}{1-r^{2}}<\frac{2 \delta}{1-r^{2}} .
$$

So for any $z \in E(a, r)$,

$$
|z-\zeta| \leq|z-a|+|a-\zeta| \leq 2 r \sqrt{s}+\delta<2 r \sqrt{\frac{2 \delta}{1-r^{2}}}+\delta
$$

Choosing $\delta$ so that $2 r \sqrt{\frac{2 \delta}{1-r^{2}}}+\delta<\epsilon$, we then have the first conclusion of the lemma.

Now suppose $a, b \in \mathbb{B}_{n}$ so that $|a-\zeta|<\delta$ and $a \in E(b, r)$. Then since $b \in E(a, r)$, the first conclusion of the lemma implies $|b-\zeta|<\epsilon$. 
For any $z \in \mathbb{B}_{n}$, the formula

$$
U_{z}(f)=\left(f \circ \varphi_{z}\right) k_{z}, f \in L^{2},
$$

defines a bounded operator on $L^{2}$. It is well-known that $U_{z}$ is a unitary operator with $L_{a}^{2}$ as a reducing subspace and $U_{z} T_{f} U_{z}^{*}=T_{f \circ \varphi_{z}}$ on $L_{a}^{2}$ for all $z \in \mathbb{B}_{n}$ and all $f \in L^{\infty}$. See, for example, [3, Lemmas 7 and 8].

Lemma 2.3. For any sequence $\left\{z_{m}\right\}_{m=1}^{\infty} \subset \mathbb{B}_{n}$ with $\left|z_{m}\right| \rightarrow 1, U_{z_{m}} \rightarrow 0$ in the weak operator topology of $\mathfrak{B}\left(L_{a}^{2}\right)$.

Proof. Since $\operatorname{Span}\left(\left\{k_{z}: z \in \mathbb{B}_{n}\right\}\right)$ is dense in $L_{a}^{2}$, it suffices to show that for all $z, w$ in $\mathbb{B}_{n}$, we have $\lim _{m \rightarrow \infty}\left\langle U_{z_{m}} k_{z}, k_{w}\right\rangle=0$.

Fix such $z$ and $w$. For each $m \geq 1$,

$$
\begin{aligned}
\left\langle U_{z_{m}} k_{z}, k_{w}\right\rangle & =\left(1-|w|^{2}\right)^{(n+1) / 2}\left(U_{z_{m}} k_{z}\right)(w) \\
& =\left(1-|w|^{2}\right)^{(n+1) / 2} k_{z}\left(\varphi_{z_{m}}(w)\right) k_{z_{m}}(w) \\
& =\frac{\left(\left(1-|w|^{2}\right)\left(1-|z|^{2}\right)\left(1-\left|z_{m}\right|^{2}\right)\right)^{(n+1) / 2}}{\left(\left(1-\left\langle\varphi_{z_{m}}(w), z\right\rangle\right)\left(1-\left\langle w, z_{m}\right\rangle\right)^{n+1}\right.} .
\end{aligned}
$$

Since $\left|\left\langle\varphi_{z_{m}}(w), z\right\rangle\right| \leq|z|$ and $\left|\left\langle w, z_{m}\right\rangle\right| \leq|w|$, we obtain

$$
\left|\left\langle U_{z_{m}} k_{z}, k_{w}\right\rangle\right| \leq \frac{\left(\left(1-|w|^{2}\right)\left(1-|z|^{2}\right)\left(1-\left|z_{m}\right|^{2}\right)\right)^{(n+1) / 2}}{((1-|z|)(1-|w|))^{n+1}} .
$$

It then follows that $\lim _{m \rightarrow \infty}\left\langle U_{z_{m}} k_{z}, k_{w}\right\rangle=0$.

Lemma 2.4. Let $\left\{z_{m}\right\}_{m=1}^{\infty} \subset \mathbb{B}_{n}$ so that $\left|z_{m}\right| \rightarrow 1$ as $m \rightarrow \infty$. Let $S$ be any nonzero positive operator on $L_{a}^{2}$. Suppose $A=\sum_{m=1}^{\infty} U_{z_{m}} S U_{z_{m}}^{*}$ exists in the strong operator topology and is a bounded operator on $L_{a}^{2}$. Then there is a constant $c>0$ and an $f \in L_{a}^{2}$ so that $\left\|A U_{z_{m}} f\right\| \geq c>0$ for all $m$.

Proof. Since $S$ is non-zero and positive, there is an $f \in L_{a}^{2}$ with $\|f\|=1$ so that $\langle S f, f\rangle>0$. For each $m \geq 1$,

$$
\begin{aligned}
\left\langle A U_{z_{m}} f, U_{z_{m}} f\right\rangle & \geq\left\langle U_{z_{m}} S U_{z_{m}}^{*} U_{z_{m}} f, U_{z_{m}} f\right\rangle \\
& \geq\left\langle S U_{z_{m}}^{*} U_{z_{m}} f, U_{z_{m}}^{*} U_{z_{m}} f\right\rangle \\
& \geq\langle S f, f\rangle .
\end{aligned}
$$

Since $\left\|U_{z_{m}} f\right\|=1$ it follows that $\left\|A U_{z_{m}} f\right\| \geq\langle S f, f\rangle>0$ for all $m$.

\section{BASIC RESUltS}

The first result in this section shows that for a certain class of subsets $G$ of $L^{\infty}, \mathfrak{I}(G)$ possesses a special property. This property will later help us distinguish $\Im\left(G_{1}\right)$ and $\mathfrak{I}\left(G_{2}\right)$ for $G_{1} \neq G_{2}$.

Proposition 3.1. Let $W$ be a subset of $\mathbb{B}_{n}$ and let $F=\operatorname{cl}(W) \cap \mathbb{S}_{n}$. Let $f$ be in $L^{\infty}$ so that $f$ vanishes almost everywhere in $\mathbb{B}_{n} \backslash W$. Let $g_{1}, \ldots, g_{l}$ be any functions in $L^{\infty}$. Let $\left\{z_{m}\right\}_{m=1}^{\infty}$ be any sequence in $\mathbb{B}_{n}$ so that $\left|z_{m}\right| \rightarrow 1$ and $\left|z_{m}-w\right| \geq$ $\epsilon>0$ for all $w \in F$, all $m \geq 1$, where $\epsilon$ is a fixed constant. Then the sequence $\left\{T_{f} T_{g_{1}} \cdots T_{g_{l}} U_{z_{m}}\right\}_{m=1}^{\infty}$ converges to 0 in the strong operator topology of $\mathfrak{B}\left(L_{a}^{2}\right)$. Consequently, if we set

$$
G=\left\{f \in L^{\infty}: f \text { vanishes almost everywhere in } \mathbb{B}_{n} \backslash W\right\},
$$


then for any $T \in \mathfrak{I}(G), T U_{z_{m}} \rightarrow 0$ in the strong operator topology of $\mathfrak{B}\left(L_{a}^{2}\right)$.

Proof. Let $V_{1}=\{|z| \leq 1:|z-w|<\epsilon / 3$ for some $w \in F\}$ and $V_{2}=\{|z| \leq 1$ : $|z-w|<\epsilon / 2$ for some $w \in F\}$. Let $\eta$ be a continuous function on $\overline{\mathbb{B}}_{n}$ so that $0 \leq \eta \leq 1, \eta(z)=1$ if $z \in \operatorname{cl}\left(V_{1}\right)$ and $\eta(z)=0$ if $z \notin V_{2}$. Let $Z=\operatorname{cl}(W) \cap\left(\overline{\mathbb{B}}_{n} \backslash V_{1}\right)$. Then $Z \subset \overline{\mathbb{B}}_{n}$ and $Z$ is compact with respect to the Euclidean metric. We have

$$
Z \cap \mathbb{S}_{n}=\operatorname{cl}(W) \cap \mathbb{S}_{n} \cap\left(\overline{\mathbb{B}}_{n} \backslash V_{1}\right)=F \cap\left(\overline{\mathbb{B}}_{n} \backslash V_{1}\right)=\emptyset .
$$

Thus $Z$ is a compact subset of $\mathbb{B}_{n}$. Since the function $f(1-\eta)$ vanishes almost everywhere in $\left(\mathbb{B}_{n} \backslash W\right) \cup \operatorname{cl}\left(V_{1}\right)$ which contains $\mathbb{B}_{n} \backslash Z$, the operator $T_{f(1-\eta)}$ is compact.

Since $\eta$ is continuous on $\overline{\mathbb{B}}_{n}$, the operators $T_{g} T_{1-\eta}-T_{g(1-\eta)}$ and $T_{1-\eta} T_{g}-T_{g(1-\eta)}$ are compact for all $g \in L^{\infty}$ (see [1]). So we have

$$
\begin{aligned}
T_{f} T_{g_{1}} \cdots T_{g_{l}} & =T_{f} T_{g_{1}} \cdots T_{g_{l}} T_{\eta}+T_{f} T_{g_{1}} \cdots T_{g_{l}} T_{1-\eta} \\
& =T_{f} T_{g_{1}} \cdots T_{g_{l}} T_{\eta}+T_{f(1-\eta)} T_{g_{1}} \cdots T_{g_{l}}+K_{1} \\
& =T_{f} T_{g_{1}} \cdots T_{g_{l}} T_{\eta}+K,
\end{aligned}
$$

where $K_{1}$ is a compact operator and $K=T_{f(1-\eta)} T_{g_{1}} \cdots T_{g_{l}}+K_{1}$ is also a compact operator.

For any $h \in L_{a}^{2} \cap L^{\infty}$ and any $m \geq 1$ we have

$$
\begin{aligned}
\left\|T_{\eta} U_{z_{m}} h\right\|^{2} & \leq\left\|\eta U_{z_{m}} h\right\|^{2} \\
& \leq \int_{V_{2}}\left|\left(U_{z_{m}} h\right)(z)\right|^{2} \mathrm{~d} \nu(z) \\
& =\int_{V_{2}}\left|h\left(\varphi_{z_{m}}(z)\right) k_{z_{m}}(z)\right|^{2} \mathrm{~d} \nu(z) \\
& \leq\|h\|_{\infty}^{2} \int_{V_{2}}\left|k_{z_{m}}(z)\right|^{2} \mathrm{~d} \nu(z) .
\end{aligned}
$$

Let $V_{3}=\{|z| \leq 1:|z-w|<\epsilon$ for some $w \in F\}$. Since the map $(z, w) \mapsto$ $|1-\langle z, w\rangle|$ is continuous and does not vanish on the compact set $\operatorname{cl}\left(V_{2}\right) \times\left(\overline{\mathbb{B}}_{n} \backslash V_{3}\right)$, there is a $\delta>0$ so that $|1-\langle z, w\rangle| \geq \delta$ for all $z \in \operatorname{cl}\left(V_{2}\right)$ and $w \in\left(\overline{\mathbb{B}}_{n} \backslash V_{3}\right)$.

For each $m \geq 1, z_{m} \in\left(\mathbb{B}_{n} \backslash V_{3}\right)$, so for all $z \in V_{2}$,

$$
\left|k_{z_{m}}(z)\right| \leq \frac{\left(1-\left|z_{m}\right|^{2}\right)^{(n+1) / 2}}{\left|1-\left\langle z, z_{m}\right\rangle\right|^{n+1}} \leq \frac{\left(1-\left|z_{m}\right|^{2}\right)^{(n+1) / 2}}{\delta^{n+1}} .
$$

Hence we have

$$
\left\|T_{\eta} U_{z_{m}} h\right\| \leq\|h\|_{\infty} \sqrt{\nu\left(V_{2}\right)} \frac{\left(1-\left|z_{m}\right|^{2}\right)^{(n+1) / 2}}{\delta^{n+1}} .
$$

This implies $\left\|T_{\eta} U_{z_{m}} h\right\| \rightarrow 0$ as $m \rightarrow \infty$. Since $L_{a}^{2} \cap L^{\infty}$ is dense in $L_{a}^{2}$ and $\left\|T_{\eta} U_{z_{m}}\right\| \leq\left\|T_{\eta}\right\| \leq 1$ for all $m$, we conclude that $T_{\eta} U_{z_{m}} \rightarrow 0$ in the strong operator topology of $\mathfrak{B}\left(L_{a}^{2}\right)$. So $T_{f} T_{g_{1}} \cdots T_{g_{l}} T_{\eta} U_{z_{m}} \rightarrow 0$ in the strong operator topology of $\mathfrak{B}\left(L_{a}^{2}\right)$. Also by Lemma 2.3. $U_{z_{m}} \rightarrow 0$ in the weak operator topology, so $K U_{z_{m}} \rightarrow 0$ in the strong operator topology for any compact operator $K$. Combining these facts with (3.1), we conclude that $T_{f} T_{g_{1}} \cdots T_{g_{l}} U_{z_{m}} \rightarrow 0$ in the strong operator topology of $\mathfrak{B}\left(L_{a}^{2}\right)$. 
The following proposition was proved by Suárez for the case $n=1$ (see [5. Proposition 2.9]). The case $n \geq 2$ is similar and can be proved with the same method. The point is that for all $n \geq 2$, the metric $\rho$ and the reproducing kernel functions have all the properties needed for Suárez's proof. See [2, Section 2] for more details.

Proposition 3.2. Let $0<r<1$ and $\left\{w_{m}\right\}_{m=1}^{\infty}$ be a sequence in $\mathbb{B}_{n}$ so that $E\left(w_{k}, r\right) \cap E\left(w_{l}, r\right)=\emptyset$ for all $k \neq l$. For each $m \in \mathbb{N}$, let $c_{m}^{1}, \ldots, c_{m}^{l}, a_{m}, b_{m}$, $d_{m}^{1}, \ldots, d_{m}^{k} \in L^{\infty}$ be functions of norm $\leq 1$ that vanish almost everywhere on $\mathbb{B}_{n} \backslash E\left(w_{m}, r\right)$. Then

$$
\sum_{m \in \mathbb{N}} T_{c_{m}^{1}} \cdots T_{c_{m}^{l}}\left(T_{a_{m}} T_{b_{m}}-T_{b_{m}} T_{a_{m}}\right) T_{d_{m}^{1}} \cdots T_{d_{m}^{k}}
$$

belongs to $\mathfrak{C} \mathfrak{I}\left(L^{\infty}\right)$.

Remark 3.3. In the proof of Proposition 3.2, we work only with Toeplitz operators with symbols in the set $G$ which consists of functions of the form $\sum_{m \in F} f_{m}$, where $F$ is a subset of $\mathbb{N}$ and $f$ is one of the symbols $c^{1}, \ldots, c^{l}, a, b, d^{1}, \ldots, d^{k}$. So in the conclusion of the proposition, we may replace $\mathfrak{C} \mathfrak{I}\left(L^{\infty}\right)$ by $\mathfrak{C} \mathfrak{I}(G)$.

\section{Proof of the MAIN TheOrem}

We are now ready for the proof of Theorem 1.1.

Fix $0<r<1$. Let $W_{\emptyset}=E(0, r)$. For any closed non-empty subset $F$ of $\mathbb{S}_{n}$, let

$$
W_{F}=\bigcup_{0<t<1} \bigcup_{\zeta \in F} E(t \zeta, r) \text {. }
$$

It is clear that $W_{\mathbb{S}_{n}}=\mathbb{B}_{n}$. We always have $F \subset \operatorname{cl}\left(W_{F}\right) \cap \mathbb{S}_{n}$. We will show that in fact $F=\operatorname{cl}\left(W_{F}\right) \cap \mathbb{S}_{n}$. Suppose $\zeta \in \operatorname{cl}\left(W_{F}\right) \cap \mathbb{S}_{n}$. For any $m \geq 1$, applying Lemma 2.2 with $\epsilon=m^{-1}$, we get a $\delta_{m}>0$ so that if $E(b, r) \cap V_{m} \neq \emptyset$, where $V_{m}=\left\{z \in \mathbb{B}_{n}:|z-\zeta|<\delta_{m}\right\}$, then $|b-\zeta|<m^{-1}$. Since $W_{F} \cap V_{m} \neq \emptyset$, there is $0<t_{m}<1$ and $\zeta_{m} \in F$ so that $E\left(t_{m} \zeta_{m}, r\right) \cap V_{m} \neq \emptyset$. Hence $\left|t_{m} \zeta_{m}-\zeta\right|<m^{-1}$. So $\left|t_{m} \zeta_{m}-\zeta\right| \rightarrow 0$ as $m \rightarrow \infty$. Since $t_{m}=\left|t_{m} \zeta_{m}\right| \rightarrow|\zeta|=1$ as $m \rightarrow \infty$, we get $\zeta_{m}=t_{m}^{-1}\left(t_{m} \zeta_{m}\right) \rightarrow \zeta$ in the Euclidean metric as $m \rightarrow \infty$. This implies that $\zeta \in F$. Thus, $\operatorname{cl}\left(W_{F}\right) \cap \mathbb{S}_{n} \subset F$ and hence, $\operatorname{cl}\left(W_{F}\right) \cap \mathbb{S}_{n}=F$.

Now define

$$
G_{F}=\left\{f \in L^{\infty}: f \text { vanishes almost everywhere in } \mathbb{B}_{n} \backslash W_{F}\right\} .
$$

It is clear that if $F_{1} \subset F_{2}$, then $W_{F_{1}} \subset W_{F_{2}}$; hence $G_{F_{1}} \subset G_{F_{2}}$.

Since $T_{f}$ is compact for all $f \in G_{\emptyset}, \mathfrak{I}\left(G_{\emptyset}\right)=\mathcal{K}$. Since $G_{\mathbb{S}_{n}}=L^{\infty}$, we have $\mathfrak{C} \mathfrak{I}\left(G_{\mathbb{S}_{n}} \cap C\left(\mathbb{B}_{n}\right)\right)=\mathfrak{C} \mathfrak{I}\left(L^{\infty} \cap C\left(\mathbb{B}_{n}\right)\right)=\mathfrak{T}$.

Now suppose $F_{1}$ and $F_{2}$ are two closed subsets of $\mathbb{S}_{n}$ so that $F_{2} \backslash F_{1} \neq \emptyset$. Let $\zeta \in F_{2} \backslash F_{1}$. From Lemma 2.1 there is a sequence $\left\{t_{m}\right\}_{m=1}^{\infty} \subset(0,1)$ with $t_{m} \uparrow 1$ and $E\left(t_{k} \zeta, r\right) \cap E\left(t_{l} \zeta, r\right)=\emptyset$ for all $k \neq l$. Let $z_{m}=t_{m} \zeta$ for all $m \geq 1$. Since $\left|z_{m}-\zeta\right| \rightarrow 0$ and $\zeta \notin F_{1}$, which is a closed subset of $\mathbb{S}_{n}$, there is an $\epsilon>0$ so that $\left|z_{m}-w\right| \geq \epsilon$ for all $w \in F_{1}$, all $m \geq 1$. Since $\operatorname{cl}\left(W_{F_{1}}\right) \cap \mathbb{S}_{n}=F_{1}$, Proposition 3.1 shows that $T U_{z_{m}} \rightarrow 0$ in the strong operator topology for all $T \in \mathfrak{I}\left(G_{F_{1}}\right)$.

Take $f$ to be any continuous function supported in $E(0, r)$ such that $\left[T_{f}, T_{\bar{f}}\right] \neq 0$. Any function of the form $f(z)=z_{1} \eta(|z| / r)$ where $\eta$ is non-negative, continuous and 
supported in $[0,1]$ with $\|\eta\|_{\infty}>0$ will work. Let $S=\left[T_{f}, T_{\bar{f}}\right]^{2}$, then $S$ is a non-zero, positive operator on $L_{a}^{2}$. Define

$$
T=\sum_{m=1}^{\infty} U_{z_{m}} S U_{z_{m}}^{*}
$$

By Lemma 2.4, there is a constant $c>0$ and an $h \in L_{a}^{2}$ so that $\left\|T U_{z_{m}} h\right\| \geq c$ for all $m$. This implies that $T$ is not in $\mathfrak{I}\left(G_{F_{1}}\right)$.

For each $m$,

$$
\begin{aligned}
U_{z_{m}}\left[T_{f}, T_{\bar{f}}\right] U_{z_{m}}^{*} & =U_{z_{m}} T_{f} T_{\bar{f}} U_{z_{m}}^{*}-U_{z_{m}} T_{\bar{f}} T_{f} U_{z_{m}}^{*} \\
& =T_{f \circ \varphi_{z_{m}}} T_{\bar{f} \circ \varphi_{z_{m}}}-T_{\bar{f} \circ \varphi_{z_{m}}} T_{f \circ \varphi_{z_{m}}} \\
& =\left[T_{f \circ \varphi_{z_{m}}}, T_{\bar{f} \circ \varphi_{z_{m}}}\right] .
\end{aligned}
$$

So $U_{z_{m}} S U_{z_{m}}^{*}=\left[T_{f \circ \varphi_{z_{m}}}, T_{\bar{f} \circ \varphi_{z_{m}}}\right]^{2}$. Hence $T=\sum_{m=1}^{\infty}\left[T_{f \circ \varphi_{z_{m}}}, T_{\bar{f} \circ \varphi_{z_{m}}}\right]^{2}$.

Since each $f \circ \varphi_{z_{m}}$ is continuous and supported in $\left\{w \in \mathbb{B}_{n}:\left|\varphi_{z_{m}}(w)\right|<r\right\}=$ $E\left(z_{m}, r\right)$ and $E\left(z_{k}, r\right) \cap E\left(z_{l}, r\right)=\emptyset$ for all $k \neq l$, Proposition 3.2 and Remark 3.3 show that $T \in \mathfrak{C} \mathfrak{I}\left(G_{F_{2}} \cap C\left(\mathbb{B}_{n}\right)\right)$. So $T \in \mathfrak{C} \mathfrak{I}\left(G_{F_{2}} \cap C\left(\mathbb{B}_{n}\right)\right) \backslash \mathfrak{I}\left(G_{F_{1}}\right)$.

\section{ACKNOWLEDGEMENTS}

This work was done when the author was a graduate student at the State University of New York at Buffalo.

\section{REFERENCES}

[1] Lewis A. Coburn, Singular integral operators and Toeplitz operators on odd spheres, Indiana Univ. Math. J. 23 (1973/74), 433-439. MR0322595 (48:957)

[2] Trieu Le, On the commutator ideal of the Toeplitz algebra on the Bergman space of the unit ball in $\mathbb{C}^{n}$, J. Operator Theory, to appear.

[3] Young J. Lee, Pluriharmonic symbols of commuting Toeplitz type operators on the weighted Bergman spaces, Canad. Math. Bull. 41 (1998), no. 2, 129-136. MR1624149 (99b:47035)

[4] Walter Rudin, Function theory in the unit ball of $\mathbf{C}^{n}$, Grundlehren der Mathematischen Wissenschaften [Fundamental Principles of Mathematical Science], vol. 241, Springer-Verlag, New York, 1980. MR601594 (82i:32002)

[5] Daniel Suárez, The Toeplitz algebra on the Bergman space coincides with its commutator ideal, J. Operator Theory 51 (2004), no. 1, 105-114. MR2055807 (2005b:47060)

Department of Pure Mathematics, University of Waterloo, Waterloo, Ontario, CANAdA N2L 3G1

E-mail address: trieu.le@utoronto.edu 